DOI: $10.12957 /$ demetra.2017.26928

\title{
Conflitos de interesses nas estratégias da indústria alimentícia para aumento do consumo de alimentos ultraprocessados e os efeitos sobre a saúde da população brasileira
}

\section{Conflicts of interest of the strategy of food industry to increase the consumption of ultra- processed foods and the health effects of the Brazilian population}

\author{
Simone Caivano ${ }^{1}$ \\ Renata Fagundes Lopes ${ }^{2}$ \\ Ana Lydia Sawaya ${ }^{3,4}$ \\ Semíramis Martins Álvares Domene ${ }^{4,5}$ \\ Paula Andrea Martins ${ }^{6}$ \\ 1 Universidade Federal de São Paulo, \\ Departamento de Políiticas Públicas e Saúde \\ Coletiva, Programa de Pós-graduação \\ Interdisciplinar em Ciências da Saúde. \\ Santos-SP, Brasil. \\ ${ }^{2}$ Universidade Federal de São Paulo, \\ Departamento de Ciências do Movimento \\ Humano, Programa de Pós-graduação em \\ Alimentos, Nutrição e Saúde. Santos-SP, Brasil. \\ ${ }^{3}$ Universidade Federal de São Paulo, \\ Departamento de Fisiologia, Fisiologia da \\ Nutrição. Santos-SP, Brasil. \\ ${ }^{4}$ Universidade de São Paulo, Instituto de Estudos \\ Avançados, Grupo de Estudos e Pesquisa Nutrição \\ e Pobreza. São Paulo-SP, Brasil. \\ ${ }^{5}$ Universidade Federal de São Paulo, \\ Departamento de Políticas Públicas e Saúde \\ Coletiva. Santos-SP, Brasil. \\ ${ }^{6}$ Universidade Federal de São Paulo, \\ Departamento de Ciências do Movimento \\ Humano. Santos-SP, Brasil. \\ Correspondência / Correspondence \\ Simone Caivano \\ E-mail:simone.caivano@hotmail.com
}

\section{Resumo}

As doenças crônicas não transmissíveis, em 2010, foram responsáveis por mais de 18 milhões de mortes no mundo; em 2014, a prevalência do excesso de peso representou 2,1 bilhões. No Brasil, o excesso de peso afeta $51 \%$ da população. Existem muitas evidências de que os alimentos ultraprocessados (biscoitos recheados, salgadinhos de pacote, refrigerantes e fast food) colaboram para essa epidemia por apresentarem combinações de ingredientes que os tornam altamente palatáveis (açúcar, sal, gordura e aditivos químicos) e que prejudicam os mecanismos de controle do apetite. Os principais fatores que contribuíram para o aumento no consumo desses alimentos foram o fortalecimento da economia, elevação do poder de compra, campanhas de marketing, bem como comodidade, conveniência e seu menor custo. A indústria de alimentos tem utilizado estratégias de formação de opinião, como a exposição no espaço midiático com campanhas maciças para a promoção desses produtos e ações com foco em profissionais da saúde por meio do patrocínio de eventos científicos e de organizações não governamentais. Os conflitos de interesse na produção científica são crescentes e a análise de estudos financiados pela indústria tem mostrado que os desfechos são muito frequentemente favoráveis ao patrocinador do estudo. Este ensaio tem o objetivo de discutir a influência da indústria alimentícia no aumento do consumo de alimentos ultraprocessados e o efeito sobre a saúde da população brasileira. Pretende-se contribuir para o processo de transformação de práticas e princípios, bem como para uma ampliação dessa 
discussão com transparência e equidade, a fim de manter o interesse da população brasileira em primeiro lugar.

Palavras-chave: Políticas Públicas. Obesidade. Consumo de Alimentos. Alimentação Saudável.

\section{Abstract}

Noncommunicable chronic diseases in 2010, accounted for over 18 million deaths worldwide; in 2014, the prevalence of overweight represented 2.1 billion. In Brazil, overweight affects $51 \%$ of the population. Ultra-processed foods as sandwich cookies, pack snacks, soft drinks and fast food, collaborate to this epidemic by presenting combinations of ingredients that make them highly palatable (sugar, salt, fat and chemical additives) and damage mechanisms of control of appetite. The main factors that contributed to the increase in the consumption of these foods were the strengthening of the economy, the increase in purchasing power, marketing campaigns, as well as the comfort, convenience and its lower cost. The food industry has used opinion formation strategies, such as exposure in the media with massive campaigns to promote these products and initiatives focused on health professionals through sponsorship of scientific events and non-governmental organizations. Conflicts of interest in scientific production are increasing and analysis of studies funded by industry has shown that the outcomes are favorable to the study sponsor. This trial aimed to discuss the influence of the food industry in the increased consumption of ultra-processed food and the effects on health of the Brazilian population. Thus, ther may be a contribution to the process in the transformation of practices and principles, as well as an extension of this discussion with transparent and fair discussion, keeping the interest of the Brazilian population first.

Keywords: Public Policies. Obesity. Food Consumption. Healthy Eating. 


\section{Introdução}

As sociedades contemporâneas vêm enfrentando um quadro epidemiológico caracterizado por altas prevalências de Doenças Crônicas Não Transmissíveis (DCNT). Em 2010, doenças como hipertensão, obesidade, diabetes e dislipidemias foram responsáveis por mais de 18 milhões de mortes no mundo. ${ }^{1}$ A incidência de obesidade correspondeu a 3,4 milhões de indivíduos em 2010, aumentando para 2,1 bilhões em 2014, em adultos. ${ }^{2}$ Em crianças e adolescentes, nos países em desenvolvimento, ocorreu um expressivo crescimento da prevalência de excesso de peso nesse período (de 8,1 a 13,4\%); em países desenvolvidos esta prevalência aumentou de 22,6 a 23,8\%. ${ }^{3}$

No Brasil, em 2007, as DCNTs foram responsáveis por $72 \%$ das mortes; ${ }^{4}$ a prevalência para o excesso de peso em adultos foi de 50,1\%, e para obesidade, 12,4\%; em crianças, 33,5\% e 14,3\%; e em adolescentes, 20,5\% e 4,9\%, respectivamente. ${ }^{5} \mathrm{Em}$ 2008, a prevalência de DCNT foi de 31,3\%, incluindo todas as faixas etárias, sendo a hipertensão responsável por 13,97\%, acometendo 57,15\% de adultos entre 70 e 79 anos; doenças do coração, 3,97\% e diabetes, 3,59\%, com uma prevalência maior a partir de 50 anos (cerca de $8 \%$ a $25 \%$ ), e câncer, $0,56 \% .{ }^{6} \mathrm{O}$ sistema público brasileiro investiu cerca de US $\$ 270$ milhões em 2011 com o cuidado da obesidade e suas comorbidades. ${ }^{7}$ Tabagismo, excesso de bebida alcoólica, inatividade física e hábitos alimentares inadequados são os principais fatores de risco para o desenvolvimento das DCNTs. ${ }^{8}$

Existem muitas evidências de que o consumo de produtos alimentares ultraprocessados tem impacto no aumento da prevalência das DCNTs. ${ }^{9-11}$ Alimentos como biscoitos recheados, salgadinhos de pacote, refrigerantes e fast food, que são classificados como ultraprocessados, ${ }^{11}$ fazem parte dos padrões alimentares relacionados a essa epidemia ${ }^{1,12}$ tendo em vista os principais ingredientes utilizados para sua produção (açúcar, sal, gordura e aditivos químicos). Estes componentes são de baixo custo, o que favorece sua produção, mas acarretam alterações nos mecanismos de controle do apetite, além de serem empregados em produtos altamente palatáveis, ${ }^{13}$ que, em sua maioria, apresentam baixa qualidade nutricional. Relatório recente da Organização Mundial da Saúde (2015) alerta que muitas vezes esses produtos alimentares são apresentados à população como saudáveis. ${ }^{12}$ Dessa forma, este ensaio teve o objetivo de discutir a influência da indústria alimentícia no aumento do consumo de alimentos ultraprocessados e o efeito sobre a saúde da população brasileira.

\section{0 papel de políticas econômicas e estratégias de comercialização sobre 0 aumento do consumo de alimentos ultraprocessados}

Uma razão importante para o aumento na aquisição, distribuição e abastecimento desses produtos são as políticas econômicas que favorecem o fluxo do capital internacional, a entrada transnacional em mercados internos e a desregulamentação do mercado desde 1980. Há uma correlação positiva entre a desregulamentação do mercado e o aumento na venda de produtos 
ultraprocessados $(\mathrm{R} 2=0,48 ; \mathrm{p}<0,001) .{ }^{12}$ No Reino Unido, Canadá e Estados Unidos, a aquisição de alimentos ultraprocessados nas últimas duas décadas foi responsável por $60 \%$ do total de energia consumida; no Brasil, este consumo está em $28 \% .{ }^{13}$ As vendas dessa categoria de alimentos aumentaram 43,7\% de 2000 para 2013, ocupando 22,3\% do total de vendas na América do Norte (Estados Unidos e Canadá) e 16,3\% na América Latina. No Brasil, o consumo de bebidas e alimentos ultraprocessados no ano 2000 foi de $86 \mathrm{~kg}$ per capita, aumentando para 112,3 $\mathrm{kg}$ per capita em 2013, apresentando um crescimento de $2,1 \%$ ao ano.

A comercialização desses alimentos é feita por grandes grupos econômicos que monopolizam o mercado. Os principais fatores que contribuíram para o aumento no consumo desses alimentos foram o fortalecimento da economia, a elevação do poder de compra, as campanhas de marketing, bem como a comodidade, a conveniência e seu menor custo. Campanhas de marketing maciças e agressivas também colaboram para o aumento na aquisição de alimentos ultraprocessados prontos para consumo, a partir da criação de necessidades no consumidor, da falta de tempo dedicada ao preparo dos alimentos, da comodidade, praticidade e conveniência trazidas por esses produtos. ${ }^{1}$ Motivações de escolha associadas à subjetividade do sujeito, igualmente influenciadas por estes mecanismos, mas também decorrentes de dimensões históricas e culturais, não são o foco de abordagem deste ensaio.

Identificam os cinco principais atores que têm impacto importante nesse cenário: as grandes indústrias transnacionais de alimentos, os profissionais da saúde, as associações e entidades da sociedade civil, os gestores públicos e a mídia. Outro aspecto que levou ao aumento da aquisição de alimentos ultraprocessados foi a estratégia da indústria para estabelecer relações com estes atores e elevar sua influência, a partir do financiamento de atividades acadêmicas, governamentais, entre outras, por meio de parcerias público-privadas e sua influência no aumento da aquisição de alimentos ultraprocessados. ${ }^{14}$

\section{Indústria de alimentos e bebidas ultraprocessados como parte atuante na promoção de alimentação saudável?}

Em publicação de 2005, Verduin et al., ${ }^{15}$ reconhecidos colaboradores de uma indústria de alimentos denominada ConAgra Foods Inc., mencionam que a indústria de alimentos poderia contribuir para o estado nutricional da população por meio da realização de ações diretas com os consumidores, no auxílio das escolhas alimentares saudáveis obtidas pela clareza e informação nutricional contida nos rótulos, na participação da educação alimentar e nutricional nas escolas para aumentar a conscientização e no oferecimento de produtos que atendam às expectativas do

consumidor no gosto, qualidade e preço. Entretanto, Ludwig \& Nestle, em $2008,{ }^{16}$ apresentam 
alguns exemplos indicativos de contradições nas ações realizadas pela indústria de alimentos ultraprocessados:

- o McDonald's faz uso de gorduras trans nas suas preparações, além de vender alimentos não saudáveis para as crianças vinculados a brinquedos e jogos;

- a Kraft vende produtos não saudáveis para crianças, como biscoitos, catchup, mostarda, maionese, chocolates, balas, gomas de mascar, entre outros;

- o Center for Consumer Freedom $(C C F)$, que seria um canal direto da indústria com o consumidor, utilizado pelos americanos, é financiado por empresas como Coca-Cola, Cargill, Tyson Foods e Wendy;

- eventos patrocinados que promovam a diversão, o movimento corporal e os jogos com crianças e seus familiares para incentivar uma vida saudável são ações que contribuem para desviar o olhar do consumidor em relação à qualidade nutricional dos próprios produtos.

Esses autores afirmam também que, em uma economia orientada para o mercado, a indústria tende a agir no interesse da maximização de lucros. Por isso, acreditam que ações que contrariam seu modelo de negócio podem implicar em menor crescimento. ${ }^{16}$

Segundo Yatch et al., ${ }^{17}$ membros da Pepsico, em artigo publicado em 2010, da mesma forma que Verduin et al., ${ }^{15}$ relataram as iniciativas da indústria que, segundo eles, poderiam auxiliar no combate à fome e desnutrição:

- investimento na agricultura, especialmente nos pequenos agricultores por meio do fornecimento de sementes de maior qualidade;

- provisionamento de microcrédito para os agricultores - com o objetivo de melhorar a acessibilidade aos fertilizantes e a eficiência dos sistemas de irrigação;

- auxílio na expansão de recursos básicos (distribuição e controle de qualidade) das corporações;

- maior apoio à fortificação de alimentos consumidos rotineiramente;

- aumento do portfólio de alimentos para a alimentação complementar direcionados à desnutrição;

- criação de modelos de negócios sociais inovadores para combater a desnutrição e a obesidade (negócio sustentável);

- investimento no desenvolvimento da ciência da nutrição, especialmente em países em desenvolvimento;

- reformulação de alimentos ditos como nutritivos por serem de baixo valor energético e de baixo custo. 
Entretanto, no ano seguinte (2011), Hawkes \& Buse ${ }^{18}$ levantaram sérios questionamentos sobre a eficácia de parcerias público-privadas ou a contribuição da indústria de alimentos ultraprocessados em atividades de promoção de alimentação saudável. Segundo esses autores, para que parcerias público-privadas com a indústria de alimentos sejam benéficas à sociedade, é preciso que o interesse público venha em primeiro lugar. Mais recentemente, em 2013, Moodie et al., ${ }^{1}$ e uma publicação da Organização Mundial da Saúde, em 2015, ${ }^{12}$ declararam que não há evidência que apoie a eficácia ou a segurança da parceria público-privada com a indústria de alimentos.

\section{Difícil trajetória da regulamentação da publicidade de alimentos ultraprocessados no Brasil}

A mídia televisiva influencia de forma negativa as preferências alimentares, principalmente o público mais vulnerável, como é o caso das crianças. ${ }^{14,16}$ No desenvolvimento das campanhas de marketing, as estratégias de venda são baseadas em técnicas que incorporam os mais recentes conhecimentos de comportamento de acordo com a psicanálise. Portanto, o marketing e a publicidade de alimentos são capazes de explorar crenças, desejos e ilusões, prejudicando as decisões racionais e de autocontrole. ${ }^{12}$

O papel regulador do Estado incide sobre o estabelecimento de padrões seguros para alimentação e nutrição, a proibição da comercialização de alimentos direcionada para crianças e a regulamentação da propaganda e publicidade de alimentos. Em muitos países, a regulamentação é ativa, seja proibindo a propaganda de produtos considerados não saudáveis, restringindo o horário e o local de sua veiculação, seja proibindo inteiramente qualquer publicidade dirigida a crianças. ${ }^{14,15}$. A Noruega e a Suíça, por exemplo, proíbem comercial de televisão voltado para crianças menores de 12 anos de idade. Da mesma forma, a Austrália também restringe a quantidade e a frequência de peças publicitárias durante a programação infantil; enquanto a Áustria e a Bélgica proíbem comerciais antes e depois de programas infantis. Por fim, a Itália apresenta um código de autorregulamentação. ${ }^{19}$ Muito embora, no Brasil, o Código de Defesa do Consumidor ${ }^{20}$ afirme que publicidade abusiva é caracterizada quando se vale da "falta de julgamento e experiência da criança", e que o Conselho Nacional dos Direitos da Criança e do Adolescente, por meio da resolução 163, de 13 de março de 2014, ${ }^{21}$ disponha "sobre a abusividade do direcionamento de publicidade e de comunicação mercadológica à criança e adolescente”, o País está muito atrás de outras nações, dada a falta de regulamentação para a publicidade de alimentos; exceção vigente é a proibição de qualquer tipo de propaganda de substitutos de leite materno. ${ }^{13}$ Em março de 2016, o Superior Tribunal de Justiça "considerou abusiva e proibiu uma campanha publicitária dirigida ao público infantil"; esta ação, relativa a uma campanha publicitária em especial, constitui precedente que poderá impactar em outros casos similares. ${ }^{22}$ 
A Constituição de 1988 estabelece que o Brasil é uma democracia participativa e, portanto, as parcerias em todos os níveis entre governo e sociedade civil devem ser consideradas como ações relevantes e importantes para o crescimento do país. ${ }^{13}$ É incontestável, porém, a importância de se regulamentar as parcerias entre indústrias produtoras de alimentos e bebidas para a garantia da saúde da população brasileira e a necessidade do aumento do poder e controle do governo sob a sociedade. ${ }^{14}$ Contudo, Henriques et al. acrescentam que as medidas que foram encaminhadas com o objetivo de regulamentação dessas atividades não foram, até o momento, contempladas por haver um grande conflito de interesse estabelecido entre a indústria de alimentos e setores do governo brasileiro. Entre tantos argumentos apresentados pelo setor, destacam-se os princípios da "liberdade de expressão", "liberdade individual" e "liberdade de escolha". 14,15 Essas afirmações, porém, desconsideram as evidências com sustentação científica que comprovam o poder de persuasão das estratégias de marketing, assim como aumento excessivo da palatabilidade, que pode gerar vício alimentar e descontrole do apetite.

\section{Conflito de interesse nas relaçóes da indústria de alimentos ultraprocessados e os profissionais da saúde no Brasil}

As associações e entidades de nutrição são órgãos constituídos por profissionais da saúde capacitados para identificar características de qualidade nutricional dos alimentos. Portanto, ao firmarem parceria com o setor produtor de alimentos ultraprocessados, estes órgãos conferem legitimidade aos produtos e sustentam a percepção de segurança por parte do consumidor, tendo em vista que seus efeitos negativos não são expostos. ${ }^{23-26} \mathrm{O}$ vínculo entre profissionais da saúde e a indústria indica endosso dos produtos, atribui emoção positiva à marca, bem como amplia as chances de fidelização dos consumidores, o que reflete diretamente no aumento das vendas. ${ }^{23}$

Outra influência causada pela aliança entre indústria de alimentos ultraprocessados e profissionais da saúde se dá por meio da divulgação de artigos científicos patrocinados: estudos internacionais revelaram que essas publicações apresentam desfechos favoráveis em quatro a oito mais vezes quando comparados às publicações não patrocinadas pela indústria. ${ }^{15}$ Pesquisas científicas patrocinadas pelo setor produtivo com interesse comercial nos resultados podem trazer resultados insuficientes para a abordagem adequada do problema, ou seja, pode haver omissão sobre as evidências associadas a risco ou prejuízo. ${ }^{25}$

Outra situação de claro conflito de interesses é ilustrada pelo financiamento a congressos nacionais e internacionais e a palestras inseridas na programação científica dos eventos; além do envolvimento financeiro, a indústria de alimentos ultraprocessados também oferece viagens e refeições em restaurantes de alto custo. ${ }^{26} \mathrm{O}$ fornecimento de brindes aos profissionais é uma forma de garantir que os produtos de dada empresa sejam indicados aos pacientes. ${ }^{25}$ 
É importante refletir também sobre o patrocínio realizado pela indústria de alimentos a eventos mundiais, tais como Copa do Mundo e Olimpíadas, principalmente se as corporações tiverem práticas, ações políticas ou produtos que colidam com os objetivos das ações de saúde pública, como, por exemplo, a presença da Coca-Cola e do McDonald’s na Copa do Mundo realizada em 2014, e também as Olimpíadas em 2016, ambas no Brasil. É importante ressaltar que a indústria se faz presente nas reuniões, distribuindo produtos e fazendo propaganda. ${ }^{27}$

A comunidade científica opera sua produção segundo diretrizes e princípios desde a concepção de um problema até a divulgação dos achados na forma de artigos ou comunicações em eventos; assim, o esforço científico deve procurar a verdade sobre o objeto de estudo de forma independente de interesses financeiros que possam não promover bem-estar social. ${ }^{28}$

\section{Avanços nas ações voltadas à alimentação saudável}

É relevante esclarecer o papel da indústria de alimentos ultraprocessados em relação aos segmentos-chave da sociedade. Algumas associações já sugerem a criação de um código de conduta: a indústria deve encontrar formas criativas para satisfazer as necessidades reais dos consumidores, fornecendo um alimento saudável, em porções reduzidas, econômico, conveniente e de qualidade, bem como deve afastar-se das atividades científicas e profissionais dos profissionais da saúde, sem nenhuma forma de constrangimento, associação de imagem ou marca, ou participação com poder decisório sobre o escopo de um estudo ou evento científico. ${ }^{14,15}$

A companhia britânica McKinsey² elaborou relatório com cerca de 70 experiências voltadas à prevenção da obesidade com mais de 600 artigos científicos referenciados. Entre os tópicos apresentados, chama a atenção a criação de um programa abrangente, que envolve gestores públicos, setor alimentício, educadores e profissionais da saúde em ações de intervenção na mudança de comportamento. Os autores desse relatório previram uma diminuição de $20 \%$ no sobrepeso e obesidade com a implementação conjunta destas ações; o relatório aponta ainda que, se fossem realizadas ações de educação alimentar e nutricional juntamente com as ações de melhorias no ambiente alimentar, seria possível reduzir os custos de intervenção para combate à obesidade em $67 \%$.

A Organização Panamericana de Saúde (OPAS) reforça o papel da dieta como determinante principal, além de políticas de comércio e agricultura, para o enfrentamento do atual cenário de adoecimento crescente da população mundial. Em outubro de 2014, membros da OPAS aprovaram o Plano de ação contra a obesidade infantil para os anos de 2014 a 2019; entre suas recomendações, estão as políticas fiscais e os incentivos para o aumento da produção e consumo de alimentos saudáveis; a regulamentação da propaganda de alimentos; uma melhor rotulagem de produtos alimentares e bebidas processadas; e melhoria da alimentação escolar e aumento 
da atividade física em escolares. Além dessas diretrizes, está a ação sinérgica e coerente entre o governo, a comunidade científica, organizações da sociedade civil, mídia e setor privado para implementar acordos políticos. ${ }^{12,29}$

Outra medida importante trata da estruturação dos subsídios agrícolas para apoiar a saúde pública e não apenas os produtores de commodities, ${ }^{16}$ associada a uma regulamentação do mercado e das medidas fiscais que não favoreçam as grandes indústrias de alimentos. São ações integrantes deste plano estrutural: estabelecer limites de concentração de terras, melhorar a disponibilidade de alimentos frescos e de origem local a fim de favorecer a agricultura familiar e promover e fortalecer a preparação de alimentos e a habilidade culinária. ${ }^{12}$

São indispensáveis ações do Estado e da sociedade civil para incentivo ao consumo de alimentos regionais, o desenvolvimento de estratégias a fim de reduzir a presença de alimentos industrializados com baixa densidade nutricional e alta densidade energética na rotina alimentar, a promoção de atividade física em ambientes urbanos seguros, bem como espaços de conveniência e meios de transporte coletivos de qualidade que possibilitem hábitos de vida sustentáveis. ${ }^{11}$ A publicação do Guia Alimentar para a População Brasileira, ${ }^{11}$ no segundo semestre de 2014, representou avanço frente às diretrizes sobre alimentação saudável para a população brasileira, ao propor a classificação dos alimentos com base no grau de processamento e ao valorizar a escolha por alimentos in natura e minimamente processados; a publicação é um convite para que a população tenha um olhar mais crítico em relação às informações que recebe sobre alimentação. ${ }^{11}$

Entende-se que controle e equilíbrio são duas palavras imperiosas para alinhar os interesses financeiros da indústria de alimentos com os objetivos de saúde pública, ${ }^{16}$ da mesma maneira que o empenho e investimento do governo. ${ }^{12}$ Essa discussão é chamada de "Ciência do efeito do comportamento corporativo na saúde", uma área emergente de saúde pública e que precisa ser desenvolvida substancialmente. ${ }^{1}$

\section{Considerações finais}

Vivemos um cenário de alta prevalência de doenças crônicas, tendo como uma das principais causas o consumo de alimentos ultraprocessados, que é favorecido pelo fluxo de capital, bem como pelo planejamento de marketing e demais ações realizadas pela indústria associadas aos profissionais da saúde.

Mesmo que muitos movimentos já estejam sendo realizados a fim de promover uma alimentação saudável, é necessário que haja transformação de práticas e princípios e que essa discussão se torne cada vez mais pública para que haja transparência e equidade. Todas as ações devem ser realizadas de acordo com os princípios de justiça social, sensibilidade cultural, viabilidade econômica e sustentabilidade ambiental. 


\section{Colaboradores}

Caivano S, Lopes RF, Sawaya AL, Domene SMA e Martins PA participaram da concepção do trabalho, redação e revisão crítica do artigo e aprovação da versão a ser publicada.

Conflito de Interesses: Os autores declaram não haver conflito de interesses.

\section{Agradecimentos}

Aos membros do grupo de pesquisa Epidemiologia Nutricional da Universidade Federal de São Paulo (UNIFESP) - campus Baixada Santista, ao Instituto de Estudos Avançados da Universidade de São Paulo e aos organizadores do IV Seminário Integração Serviço-Pesquisa. Estes não fazem parte da autoria e também não são financiadores deste manuscrito.

\section{Referências}

1. Moodie R, Stuckler D, Monteiro C, Sheron N, Neal B, Thamarangsi T, et al. Profits and pandemics: prevention of harmful effects of tobacco, alcohol, and ultra-processed food and drink industries. The Lancet. 2013;381(9867):670-9.

2. Dobbs R, Sawers C, Thompson F, Manyika J, Woetzel J, Child P. Overcoming obesity: an initial economic analysis. McKinsey Global Institute. 2014;1-105.

3. Ng M, Fleming T, Robinson M, Thomson B, Graetz N, Margono C, et al. Global, regional, and national prevalence of overweight and obesity in children and adults during 1980-2013: a systematic analysis for the Global Burden of Disease Study 2013. The Lancet. 2014;384(9945):766-81.

4. Schmidt M, Duncan B, Silva G, Menezes A, Monteiro C, Barreto S. Doenças crônicas não transmissíveis no Brasil: carga e desafios atuais. The Lancet. 2011;61-74.

5. Instituto Brasileiro de Geografia e Estatística. IBGE. Antropometria e Estado Nutricional de Crianças, Adolescentes e Adultos no Brasil. Pesquisa de Orçamentos Familiares 2008-2009. Rio de Janeiro: IBGE; 2010.

6. de Azevedo Barros MB, Francisco PMSB, Zanchetta LM, César CLG. Tendências das desigualdades sociais e demográficas na prevalência de doenças crônicas no Brasil, PNAD: 2003-2008. Revista Ciência \& Saúde Coletiva. 2011;16(9):3755-3768.

7. de Oliveira ML, Santos LMP, da Silva EN. Direct healthcare cost of obesity in Brazil: An application of the cost-of-Illness method from the perspective of the public health system in 2011. PloSone. 2015;10(4).

8. Wang Y, Beydoun MA, Liang L, Caballero B, Kumanyika SK. Will all Americans become overweight or obese? Estimating the progression and cost of the US obesity epidemic. Obesity. 2008;16(10):2323-30. 
9. WHO. World Health Organization. Assessing national capacity for the prevention and control of noncommunicable diseases: report of the 2010 global survey. Geneva: World Health Organization. 2012.

10. Katz D, Meller S. Can we say what diet is best for health? Annual review of public health. 2014;35:83103. doi: 10.1146/annurev-publhealth-032013-182351.

11. Brasil. Ministério da Saúde. Secretaria de Atenção à Saúde. Departamento de Atenção Básica. Guia Alimentar para a População Brasileira. Brasília; 2014.

12. World Health Organization. WHO. Ultra-processed food and drink products in Latin America: Trends, impact on obesity, policy implications. Washington, D.C.: PAHO. Pan American Health Organization; 2015.

13. Monteiro CA, Cannon G. The impact of transnational "big food"companies on the South: a view from Brazil. PLoS Med. 2012;9(7):e1001252.

14. Henriques P, Dias PC, Burlandy L. A regulamentação da propaganda de alimentos no Brasil: convergências e conflitos de interesses. Cad Saúde Pública. 2014;30(6):1219-28.

15. Verduin P, Agarwal S, Waltman S. Solutions to obesity: perspectives from the food industry. The American journal of clinical nutrition. 2005;82(1):259S-61S.

16. Ludwig DS, Nestle M. Can the food industry play a constructive role in the obesity epidemic? JAMA. 2008;300(15):1808-11.

17. Yach D, Feldman ZA, Bradley DG, Khan M. Can the food industry help tackle the growing global burden of undernutrition? American journal of public health. 2010;100(6):974.

18. Hawkes C, Buse K. Public health sector and food industry interaction: it's time to clarify the term 'partnership'and be honest about underlying interests. The European Journal of Public Health. 2011;21(4):400-1.

19. Moura NC. Influência da mídia no comportamento alimentar de crianças e adolescentes. Segurança Alimentar e Nutricional. 2010;17(1):113-122.

20. Brasil. Lei no 8.078, de 11 de setembro de 1990. Código de Defesa do Consumidor. Diário Oficial da União 12 nov 1990.

21. Conselho Nacional dos Direitos da Criança e do Adolescente (Brasil). Resolução CONANDA no 163 de 13 de março de 2014. Dispõe sobre a abusividade de publicidade e de comunicação mercadológica à criança e ao adolescente. Diário Oficial da União 4 abr 2014.

22. Instituto Brasileiro de Defesa do Consumidor. IDEC [Internet]. STJ proibe publicidade dirigida a crianças. 2016 mar 17 [acesso 2016 mar 22]. Disponível em: http://www.idec.org.br/em-acao/emfoco/stj-proibe-publicidade-dirigida-a-criancas.

23. Freedhoff Y, Hébert PC. Partnerships between health organizations and the food industry risk derailing public health nutrition. Canadian Medical Association Journal. 2011;183(3):291-2.

24. Brownell KD, Warner KE. The perils of ignoring history: big tobacco played dirty and millions died. How similar is big food? Milbank Quarterly. 2009;87(1):259-94. 
25. Pereira TN, do Nascimento FA, Bandoni DH. Conflito de interesse na formação e prática do nutricionista: regulamentar é preciso. Revista Ciência \& Saúde Coletiva. 2015;no prelo.

26. Nestle M. Food company sponsorship of nutrition research and professional activities: a conflict of interest? Public Health Nutrition. 2001;4(05):1015-22.

27. de Sá TH. Can Coca Cola promote physical activity? The Lancet. 2014;383(9934):2041.

28. Bes-Rastrollo M, Schulze MB, Ruiz-Canela M, Martinez-Gonzalez MA. Financial conflicts of interest and reporting bias regarding the association between sugar-sweetened beverages and weight gain: a systematic review of systematic reviews. PLoS Med. 201;10(12):e1001578.

29. Pan American Health Organization. PAHO. Plan of action for the prevention of obesity in children and adolescents. In: Americas rDCtSotRCoWft, editor. Washington, D.C;2014. 